九州大学学術情報リポジトリ

Kyushu University Institutional Repository

\title{
POWERSET MONAD, FILTER MONAD AND PRIMEFILTER MONAD IN THE CATEGORY OF SETS WITH MONOID ACTIONS
}

Mizoguchi, Yoshihiro

Department of Mathematics, Kyushu University

https://doi.org/10.5109/13370

出版情報: Bulletin of informatics and cybernetics. 21 (3/4), pp.83-95, 1985-03. Research Association of Statistical Sciences

バージョン:

権利関係 : 


\title{
POWERSET MONAD, FILTER MONAD AND PRIMEFILTER MONAD IN THE CATEGORY OF SETS WITH MONOID ACTIONS
}

\author{
By
}

\section{Yoshihiro Mrzoguch *}

\begin{abstract}
We construct a powerset monad, a filter monad and a primefilter monad in the category $M$-Set of sets with $M$-actions. To investigate the categories of algebras of these monads in $M$-Set, we consider the category of complete semilattices (resp. continuous lattices, compact Hausd orff spaces) with $M$-actions. We show that if $M$ is a group, this category is isomorphic to the category of algebras of the powerset monad (resp. filter monad, primefilter monad) in $M$-Set.
\end{abstract}

\section{Introduction}

Continuous lattices were introduced by Scott [5] as semantic domains of programming languages. In denotational semantics, data types are characterized by recursive domain equations. So we can argue about semantics only in a category where these equations have solutions. The category $\mathrm{CL}$ of continuous lattices and continuous map has just this property. So the investigation of continuous lattices has made a considerable contribution to the development of the semantic theory. For example, the domain equation, $D=[D \rightarrow D]$, has only trivial solutions in the category Set of sets and maps. But in the category $\mathrm{CL}$, the equation has a non-trivial solution $D_{\infty}$ and this domain plays an important role in the theory of lambda calculus. Further, since continuous lattices have rich structures, many mathematical properties are studied. As one of them, Day [1] identified the category of the filter monad algebras in Set with the category of continuous lattices together with non-empty directed join and arbitrary meet preserving maps as morphisms.

At this point, turning round to see these things, we find these theories and domains are based on the classical logic or on the category Set. So the following ideas appear : What happens, when we can think these things on the intuitionistic logic or on a topos. If we can consider the continuous lattices in a topos, many interesting questions occur. Is there a domain equation which could be solved by classical logic but does not have solutions by intuitionistic logic? How change the solutions of a domain equations? These differences seem to be caused by an essential point of the relation between the semantic theory of programmings and logics.

\footnotetext{
* Department of Mathematics, Kyushu University 33, Fukuoka 812, Japan.
} 
With these motivations, we first begin considering continuous lattices in a kind of topoi $M$-Set. The category $M$-Set provides a rich source of examples of topoi. For example by assigning a suitable monoid to $M$, the category $M$-Set exhibits a nonclassical but bivalent topos [2]. By the results of Day we consider following problems in this paper: Can we consider the filter monad in the topos M-Set? Dose the algebra of this monad in M-Set have a rich structure as a continuous lattice has? In algebraic theory, we first consider a monad (triple) in a base category, next we define algebras by using this monad and construct a category of these algebras [3]. Answering to the first question, we change the base category Set of the filter monad to $M$-Set. In other words, we extend this algebraic theory from Set to $M$-Set. For attacking the second question, we compare the algebras of this monad with continuous lattices equipped with $M$-actions by making a functor between two categories of these objects.

At the same time, the powerset monad and the ultrafilter monad in Set are extended to monads in $M$-Set. The algebras of these monads are complete semilattices and compact Hausdorff spaces $[3,4]$. We also compare these objects equipped with $M$-actions with algebras in $M$-Set by using fucntors.

In section 2 , we tabulate some properties of the category $M$-Set, algebraic functors [6] and relations, which are fundamental concepts in this paper. In section 3, we construct a powerset monad $P_{M}$ in $M$-Set by using powerobjects and the category $M$-Set ${ }^{P}$ of $P_{M}$-algebras. On the other hand, we consider the category $M$ - $\left(\operatorname{Set}^{P}\right)$ of $P$-algebras with $M$-actions and $M$-action preserving $P$-algebra homomorphisms. In other words, objects of $M$-(Set $\left.{ }^{P}\right)$ are complete semilattices with supremum preserving $M$-actions because it is well known that $P$-algebras are complete semilattices [4]. To compare these two categories $M$-Set ${ }^{P_{M}}$ and $M$-(Set $\left.{ }^{P}\right)$, we define an algebraic functor from $M$-Set ${ }^{P_{M}}$ to $M$-(Set $\left.{ }^{P}\right)$. We show that if $M$ is a group, then it is an isomorphism of categories. That is, if $M$ is a group $P_{M}$-algebras are complete semilattices with supremum preserving $M$-actions. In section 4 , we extend the filter monad $F$ in Set to a monad in $M$-Set. As the powerobject on an $M$-set is regarded as the set of $M$-relations ( $c f . \S 2$ ), we can naturally induce an order (subset relation) on the powerobject and we can define a filter on $M$-sets by using this order. So the usual filter monad $F$ can be extended to a monad $F_{M}$ in $M$-Set naturally. For the filter monad $F_{M}$, it is shown that if $M$ is a group then the category $M$-Set ${ }^{F_{M}}$ of $F_{M}$-algebras is isomorphic to the category $M$-(Set $\left.{ }^{F}\right)$ of $F$-algebras with $M$-actions and $M$-action preserving $F$-algebra homomorphisms. By the result of Day [1] that the objects of Set $^{F}$ are continuous lattices, it follows that if $M$ is a group then $F_{M}$-algebras are continuous lattices with non-empty directed join and arbitrary meet preserving $M$-actions. In section 5 , by modifying the definitions of $\S 4$, we obtain the primefilter monad $U_{M}$ in $M$-Set. If $M$ is a group, a primefilter on an $M$-set is an ultrafilter. So the monad $U_{M}$ includes the ultrafilter monad $U$ in Set by assigning one-point-monoid 1 to $M$. And we show several properties of the monad $U_{M}$ by using the results of $\S 4$. Especially, we show that if $M$ is a group then $U_{M}$-algebras are compact Hausdorff spaces with continuous $M$-actions. 


\section{Preliminaries}

We recall some relational notations and properties.

Let $A, B$ and $C$ be sets. When $\alpha$ is a subset of $A \times B$, we call that $\alpha$ is a relation from $A$ to $B$ and denote it by $\alpha: A \neg B$. For relations $\alpha: A \neg B$ and $\beta: B \rightarrow C$, we define a composite $\beta \cdot \alpha: A \rightarrow C$ of $\alpha$ and $\beta$ by $\beta \cdot \alpha:=\{(a, c) \in A \times C \mid(a, b) \in \alpha$ and $(b, c) \in \beta$ for some $b \in B\}$. For a relation $\alpha: A \neg B$, we denote by $\alpha^{\#}: B \neg A$ the inverse relation $\alpha_{\sharp}:=\{(b, a) \in B \times A \mid(a, b) \in \alpha\}$. We identify a map $f: A \rightarrow B$ with a relation $\{(a, f(a))$ $\in A \times B \mid a \in A\}$ (the graph of $f$ ).

Let 1 be the one-point set and $A$ be a set. In this paper we identify an element $a \in A$ with a map $a: 1 \rightarrow A$ and a subset $\alpha \subset A$ with a relation $\alpha: 1 \rightarrow A$.

Let $M$ be a monoid with unit $e$. For a set $A$ a collection $\sigma=\left\{\sigma_{m}: A \rightarrow A \mid m \in M\right\}$ of maps $\sigma_{m}$ from $A$ to $A$ is called an $M$-action on the set $A$ if $\sigma$ satisfies $\sigma_{e}=1_{A}$ and $\sigma_{m} \cdot \sigma_{n}=\sigma_{m n}$ for each $m, n \in M$. An $M$-set is defined to be a pair $A=(A, \sigma)$ of set $A$ and an $M$-action $\sigma$ on $A$. For $M$-sets $\boldsymbol{A}=(A, \sigma)$ and $\boldsymbol{B}=(B, \tau)$ we call a map $\Phi: A \rightarrow B$ an $M$-map $\Phi: \boldsymbol{A} \rightarrow \boldsymbol{B}$ if $\tau_{m} \cdot \Phi=\Phi \cdot \sigma_{m}$ for each $m \in M$. The category of $M$-sets and $M$ maps is denoted by $M$-Set.

Generally, let $\mathcal{C}$ be a category. A pair $A=(A, \sigma)$ of an object $A$ of $\mathcal{C}$ and a collection $\sigma=\left\{\sigma_{m}: A \rightarrow A \mid m \in M\right\}$ of endomorphisms $\sigma_{m}$ in $\mathcal{C}$ is an M-object in $\mathcal{C}$ if it satisfies $\sigma_{e}=\mathbf{1}_{A}$ and $\sigma_{m} \cdot \sigma_{n}=\sigma_{m n}$ in $\mathcal{C}$ for each $m, n \in M$. For $M$-objects $\boldsymbol{A}=(A, \sigma)$ and $\boldsymbol{B}=(B, \tau)$ in $\mathcal{C}$ a morphism $\Phi: A \rightarrow B$ in $\mathcal{C}$ is an $M$-morphism in $\mathcal{C}$ if $\tau_{m} \cdot \Phi=\Phi \cdot \sigma_{m}$ for each $m \in M$. We denote by $M-\mathcal{C}$ the category of $M$-objects in $\mathcal{C}$ and $M$-morphisms in $C$.

We note that if $M$ is a one-point-monoid 1, then 1-Set is isomorphic to the category Set of sets and maps.

A monoid $M$ is abelian if $n m=m n$ for any $m, n \in M$. We denote by $r$ and $l$, the right multiplication and the left multiplication of $M$, respectively. That is, $r$ and $l$ are defined by $r_{m}(n)=n m=l_{n}(m)$. We note that $\boldsymbol{M}=(M, l)$ is an $\boldsymbol{M}$-set and $r_{n}: \boldsymbol{M} \rightarrow \boldsymbol{M}$ is an $M$-map for each $n \in M$. For an $M$-set $\boldsymbol{A}=(A, \sigma)$ we call a subset $\alpha$ of $A$ an $M$-subset of $\boldsymbol{A}$ if it is closed under $\sigma$. There is a one-to-one correspondence between $M$-subsets of $\boldsymbol{A}$ and mono-subobjects of $\boldsymbol{A}$ in $M$-Set. We call an $M$-subset $\alpha$ of $\boldsymbol{M} \times \boldsymbol{A}$ an $M$-relation from $M$ to $\boldsymbol{A}$ and denote it by $\alpha: \boldsymbol{M} \rightarrow \boldsymbol{A}$. The condition that $\alpha$ is an $M$-subset is translated to an equivalent relational expression $\alpha \subset \sigma_{m}^{*} \cdot \alpha \cdot l_{m}(m \in M)$. An $M$-morphism $\alpha: \boldsymbol{M} \rightarrow \boldsymbol{A}$ is naturally identified with an $M$-relation. We call a subset $\gamma: 1 \rightarrow M$ a left ideal of $\boldsymbol{M}$ if $l_{m} \cdot \gamma \subset \gamma$ for each $m \in M$. We denote by $L_{M}$ the set of all left ideals of $M$.

It is well known [2] that $M$-Set is a topos and the subobject classifier $\Omega$ in $M$-Set is $\left(L_{M}, \boldsymbol{\omega}\right)$, where $\omega_{m}(\gamma):=r_{m}^{\#} \cdot \gamma$ for $m \in M$ and $\gamma \in L_{M}$.

LEMMA 2.1. For an $M$-set $\boldsymbol{A}=(A, \sigma)$ the powerobject $\Omega^{A}$ is the set of all M-relations from $M$ to $\boldsymbol{A}$ with the $M$-action $\rho^{A}$ defined by $\rho_{m}^{A}(\alpha):=\alpha \cdot r_{m}$ for $m \in M$ and $\alpha \in \Omega^{A}$.

PROOF. The powerobject $\Omega^{A}$ is the set of all $M$-morphisms $\tilde{\alpha}: M \times A \rightarrow \Omega$ with the $M$-action $p$ defined by $p_{m}(\tilde{\alpha}):=\tilde{\alpha} \cdot\left(r_{m} \times \mathbf{1}_{A}\right)$ for $m \in M$ and $\tilde{\alpha} \in \Omega^{A} \quad[2]$. Since $\Omega$ is a subobject classifier, there is a one-to-one correspondence between $M$-morphisms $\tilde{\boldsymbol{\alpha}}: M \times A \rightarrow \Omega$ and mono-subobjects $\alpha$ of $\boldsymbol{M} \times \boldsymbol{A}$. Hence we can identity $\Omega^{A}$ with the set 
of all $\boldsymbol{M}$-relations from $\boldsymbol{M}$ to $\boldsymbol{A}$. Let $\rho^{A}$ be the $M$-action on the set of all $M$-relations induced by $p$. Then we obtain $\rho_{m}^{A}(\alpha)=\alpha \cdot r_{m}\left(=\left(r_{m} \times 1_{A}\right)^{-1}(\alpha)\right)$ for $m \in M$ and $\alpha \subseteq \Omega^{A}$ because the following diagram must be a pullback.

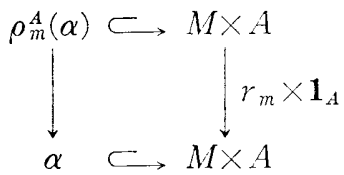

For a monad $\boldsymbol{T}=\left(T, \gamma_{\gamma}, \mu\right)$ in Set we define a monad $\overline{\boldsymbol{T}}=(\bar{T}, \bar{\gamma}, \bar{\mu})$ in $M$-Set as follows. Let $\boldsymbol{A}, \boldsymbol{B}$ be $M$-sets and $\Phi: \boldsymbol{A} \rightarrow \boldsymbol{B}$ an $M$-morphism. We define $\bar{T} \boldsymbol{A}$ by $(T A, T \sigma)$, where $(T \sigma)_{m}:=T\left(\sigma_{m}\right)$ and $\bar{T} \Phi:=T \Phi$. The natural transformations $\bar{\gamma} \boldsymbol{A}$ : $\boldsymbol{A} \rightarrow \bar{T} \boldsymbol{A}$ and $\bar{\mu} \boldsymbol{A}: \bar{T}^{2} \boldsymbol{A} \rightarrow \bar{T} \boldsymbol{A}$ are defined by $\bar{\eta} \boldsymbol{A}:=\eta \boldsymbol{A}$ and $\bar{\mu} \boldsymbol{A}:=\mu \boldsymbol{A}$, respectively. It is obvious to see that $\bar{T}$ is a monad in $M$-Set. We denote by $M$-Set ${ }^{\bar{T}}$ the category of $\bar{T}$-algebras and $\bar{T}$-morphisms [3].

Lemma 2.2. Let $\boldsymbol{A}=(A, \sigma)$ be an $M$-set and $x: T A \rightarrow A$ a map. Then the following conditions are equivalent:

(a) $x \cdot \eta A=\mathbf{1}_{A}, x \cdot \mu A=x \cdot T x$ and $\sigma_{m} \cdot x=x \cdot T \sigma_{m}$ for each $m \in M$,

(b) $((A, \sigma), x)$ is an object of $M-\operatorname{Set}^{\bar{T}}$,

(c) $((A, x), \sigma)$ is an object of $M-\left(\operatorname{Set}^{T}\right)$.

Proof. Since $(a) \leftrightarrow(c)$ and $(b) \leftrightarrow(c)$ are trivial, we only show (a) $\rightarrow$ (b). The last equation of (a) shows that $x: \bar{T} \boldsymbol{A} \rightarrow \boldsymbol{A}$ is an $M$-morphism, so the other equations of (a) imply $x \cdot \bar{\eta} \boldsymbol{A}=\mathbf{1}_{A}$ and $x \cdot \bar{\mu} \boldsymbol{A}=x \cdot \bar{T} x$ in $M$-Set. Therefore $((A, \sigma), x) \in M$-Set ${ }^{\bar{T}}$.

Lemma 2.3. Let $\boldsymbol{A}=(A, \sigma)$ and $\boldsymbol{B}=(B, \tau)$ be $M$-sets, $(\boldsymbol{A}, x)$ and $(\boldsymbol{B}, y)$ objects of $M$-Set ${ }^{\bar{T}}$ and $\Phi: A \rightarrow B$ a map. Then the followings are eqyivalent:

(a) $\Phi \cdot x=y \cdot T \Phi$ and $\Phi \cdot \sigma_{m}=\tau_{m} \cdot \Phi$ for each $m \in M$,

(b) $\Phi:((A, \sigma), x) \rightarrow((B, \tau), y)$ is a morphism in $M$-Set ${ }^{\bar{T}}$,

(c) $\Phi:((A, x), \sigma) \rightarrow((B, y), \tau)$ is a morphism in $M$-(Set $\left.{ }^{T}\right)$.

Proof. Since $(a) \leftrightarrow(c)$ and $(b) \leftrightarrow(c)$ are trivial, we only show (a) $\rightarrow$ (b). The last equation of (a) shows that $\Phi: A \rightarrow B$ is an $M$-morphism, so the first equation shows that $\Phi \cdot x=y \cdot \vec{T} \Phi$ in $M$-Set. Therefore $\Phi:(\boldsymbol{A}, x) \rightarrow(\boldsymbol{B}, y)$ is a $\bar{T}$-morphism.

By Lemma 2.2 and Lemma 2.3 we obtain the next proposition.

Proposition 2.4. $M$-Set ${ }^{\bar{T}} \cong M$-(Set $\left.{ }^{T}\right)$.

For two monads $\boldsymbol{Q}=\left(Q, \eta_{Q}, \mu_{Q}\right)$ and $\boldsymbol{R}=\left(R, \eta_{R}, \mu_{R}\right)$ in $M$-Set, a natural transformation $s: Q \rightarrow R$ is called algebraic if for each $M$-set $\boldsymbol{A}$,

$$
\left.\begin{array}{rl}
s \boldsymbol{A} \cdot \eta_{Q} \boldsymbol{A} & =\eta_{R} \boldsymbol{A}, \\
s \boldsymbol{A} \cdot \mu_{Q} \boldsymbol{A} & =\mu_{R} \boldsymbol{A} \cdot s R \boldsymbol{A} \cdot Q s \boldsymbol{A}
\end{array}\right\} .
$$

If $s: Q \rightarrow R$ is an algebraic natural transformation, then a functor $s^{*}: M-$ Set $^{R} \rightarrow M$-Set ${ }^{Q}$ is defined by $s^{*}(\boldsymbol{A}, x):=(\boldsymbol{A}, x \cdot s \boldsymbol{A})$ for $(\boldsymbol{A}, x) \in M-\operatorname{Set}^{R}$, and $s^{*} \Phi:=\Phi$ for a morphism $\Phi:(\boldsymbol{A}, x) \rightarrow(\boldsymbol{B}, y)$ in $M$-Set ${ }^{R}[6]$. The functor $s^{*}$ defined in this manner is called an algebraic functor induced by $s$.

LEMMA 2.5. If an algebraic natural transformation $s: Q \rightarrow R$ is an isomorphism, then the induced algebraic functor $s^{*}: M-\operatorname{Set}^{R} \rightarrow M-\operatorname{Set}^{Q}$ is an isomorphism of categories. 
Proof. Let $t: R \rightarrow Q$ be the inverse natural transformation of $s$. Since $t \boldsymbol{A} \cdot s \boldsymbol{A}=\mathbf{1}_{Q A}$ and $s \boldsymbol{A} \cdot t \boldsymbol{A}=\mathbf{1}_{R A}$, it easily follows from the algebraic condition (2.1) for $s$ that $\eta_{Q} \boldsymbol{A}=$ $t \boldsymbol{A} \cdot s \boldsymbol{A} \cdot \eta_{Q} \boldsymbol{A}=t \boldsymbol{A} \cdot \eta_{R} \boldsymbol{A}$ and $\mu_{Q} \boldsymbol{A} \cdot t Q \boldsymbol{A} \cdot R t \boldsymbol{A}=t \boldsymbol{A} \cdot s \boldsymbol{A} \cdot \mu_{Q} \boldsymbol{A} \cdot t Q \boldsymbol{A} \cdot R t \boldsymbol{A}=t \boldsymbol{A} \cdot \mu_{R} \boldsymbol{A} \cdot s R \boldsymbol{A} \cdot Q s \boldsymbol{A} \cdot$ $t Q \boldsymbol{A} \cdot R t \boldsymbol{A}=t \boldsymbol{A} \cdot \mu_{R} \boldsymbol{A} \cdot s R \boldsymbol{A} \cdot Q s \boldsymbol{A} \cdot Q t \boldsymbol{A} \cdot t R \boldsymbol{A}=t \boldsymbol{A} \cdot \mu_{R} \boldsymbol{A} \cdot s R \boldsymbol{A} \cdot t R \boldsymbol{A}=t \boldsymbol{A} \cdot \mu_{R} \boldsymbol{A}$. Therefore the condition (2.1) for $t$ holds. So we have an algebraic functor $t^{*}: M$-Set ${ }^{Q} \rightarrow M-$ Set $^{R}$ and it is obvious that $t^{*}$ is inverse functor of $s^{*}$.

In the rest of this paper, if no confusion occurs we omit subscripts and superscripts from natural transformations.

\section{Powerset Monad in M-Set}

In this section, we introduce a powerset monad $\boldsymbol{P}_{M}=\left(P_{M}, \eta_{M}, \mu_{M}\right)$ in $M$-Set. Next, to compare the category $M$-Set ${ }^{P_{M}}$ of $\boldsymbol{P}_{M}$-algebras with the category $M$-Set ${ }^{\bar{P}}$ of $\boldsymbol{P}$-algebras, we define an algebraic functor $s_{P}{ }^{*}: M-\operatorname{Set}^{P_{M}} \rightarrow M$-Set ${ }^{\bar{P}}$. Finally we show that if $M$ is a group, this functor $s_{P}^{*}$ is an isomorphism of categories.

Let $\boldsymbol{A}, \boldsymbol{B}$ be $M$-sets and $\Phi: \boldsymbol{A} \rightarrow \boldsymbol{B}$ an $M$-morphism. We define an endofunctor $P_{M}: M$-Set $\rightarrow M$-Set by $P_{M} A:=Q^{A}$ with an $M$-action $\rho^{A}$ and $P_{M} \Phi(\alpha):=\Phi \cdot \alpha\left(\alpha \in \Omega^{A}\right)$.

Moreover we can define natural transformations $\eta_{M}^{P}: \mathbf{1}_{M \text {-set }} \rightarrow P_{M}$ and $\mu_{M}^{P}: P_{M}^{2} \rightarrow P_{M}$ as follows.

For an $M$-set $\boldsymbol{A}=(A, \sigma), a \in \boldsymbol{A}$ and $\mathcal{A} \in P_{M}^{2} \boldsymbol{A}$,

$$
\begin{array}{lll}
(m, c) \in \eta_{M}^{P} \boldsymbol{A}(a) & \text { iff } & c=\sigma_{m}(a), \\
(m, a) \in \mu_{M}^{P} \boldsymbol{A}(\mathcal{A}) & \text { iff } & (m, \alpha) \in \mathcal{A} \text { and }(e, a) \in \alpha \text { for some } \alpha \in P_{M} \boldsymbol{A} .
\end{array}
$$

To prove exactly that $P_{M}$ defines a functor and $\eta_{M}^{P}, \mu_{M}^{P}$ define natural transformations, we must check the followings :

( i ) $P_{M} \Phi(\alpha)$ is an M-relation for $\alpha \in P_{M} A$,

(ii ) $P_{M} \Phi$ is an M-morphism,

(iii) $\eta_{M}^{P} \boldsymbol{A}(a)$ is an M-relation for $a \in \boldsymbol{A}$,

(iv) $\eta_{M}^{P} \boldsymbol{A}$ is an M-morphism,

( v ) $\eta_{M}^{P}$ is a natural transformation,

(vi) $\mu_{M}^{P} \boldsymbol{A}(\mathcal{A})$ is an $M$-relation for $\mathcal{A} \in P_{M}^{2} \boldsymbol{A}$,

(vii) $\mu_{M}^{P} \boldsymbol{A}$ is an $M$-morphism,

(viii) $\mu_{M}^{P}$ is a natural transformation.

We now verify above conditions. Let $m$ and $n$ be elements of $M$.

( i ) $\tau_{n}^{\#} \cdot P_{M} \Phi(\alpha) \cdot l_{n}=\tau_{n}^{\#} \cdot \Phi \cdot \alpha \cdot l_{n} \supset \Phi \cdot \sigma_{n}^{\#} \cdot \alpha \cdot l_{n} \supset \Phi \cdot \alpha=P_{M} \Phi(\alpha)$.

( ii ) $\left(\rho_{m}^{B} \cdot P_{M} \Phi\right)(\alpha)=\rho_{m}^{B}(\Phi \cdot \alpha)=\Phi \cdot \alpha \cdot r_{m}=P_{M} \Phi\left(\alpha \cdot r_{m}\right)=\left(P_{M} \Phi \cdot \rho_{m}^{A}\right)(\alpha)$.

(iii ) $\left(\sigma_{n} \cdot \eta \boldsymbol{A}(a)\right)(m)=\left(\sigma_{n} \cdot \sigma_{m}\right)(a)=\sigma_{n m}(a)=\left(\eta \boldsymbol{A}(a) \cdot l_{n}\right)(m)$.

(iv) Since $\left(\eta \boldsymbol{A}(a) \cdot r_{n}\right)(m)=(\eta \boldsymbol{A}(a))(m n)=\sigma_{m n}(a)=\sigma_{m} \cdot \sigma_{n}(a)=\left(\left(\eta \boldsymbol{A} \cdot \sigma_{n}\right)(a)\right)(m)$, we have $\left(\rho_{n}^{\boldsymbol{A}} \cdot \eta \boldsymbol{A}\right)(a)=\eta \boldsymbol{A}(a) \cdot r_{n}=\left(\eta \boldsymbol{A} \cdot \sigma_{n}\right)(a)$.

$(\mathrm{v})$ Since $(\Phi \cdot \eta \boldsymbol{A}(a))(m)=\left(\Phi \cdot \sigma_{m}\right)(a)=\left(\tau_{m} \cdot \Phi\right)(a)=((\eta \boldsymbol{B} \cdot \Phi)(a))(m)$, we have $\left(P_{M} \Phi \cdot \eta \boldsymbol{A}\right)(a)=\Phi \cdot \eta \boldsymbol{A}(a)=(\eta \boldsymbol{B} \cdot \Phi)(a)$.

(vi) We first show

$$
\mu \boldsymbol{A}(\mathcal{A})=\bigcup_{(n, \alpha) \in \mathcal{A}} \alpha \cdot r_{n}^{\#}
$$


If $(m, a) \in \mu \boldsymbol{A}(\mathcal{A})$ then there exist $\alpha \in P_{M} \boldsymbol{A}$ such that $(m, \alpha) \in \mathcal{A}$ and $(e, a) \in \alpha$. Therefore $(m, a) \in \alpha \cdot r_{m}^{\#} \subset \bigcup_{(n, \alpha) \in \mathcal{A}} \alpha \cdot r_{n}^{\#}$. Conversely assume $(m, a) \in \bigcup_{(n, \alpha) \in \mathcal{A}} \alpha \cdot r_{n}^{\#}$. Then we have $(m, a) \in \alpha \cdot r_{n}^{*}$ for some $(n, \alpha) \in \mathcal{A}$, and so $m=k n=l_{k}(n)$ and $(k, a) \in \alpha$ for some $k \in M$. Hence $(e, a) \in \alpha \cdot r_{k}$. As $\mathcal{A}$ is an $M$-relation, $(n, \alpha) \in \mathcal{A}$ implies $\left(l_{k}(n), \rho_{k}^{A}(\alpha)\right)=\left(m, \alpha \cdot r_{k}\right) \in \mathcal{A}$. This shows $(m, a) \in \mu A(\mathcal{A})$.

By using (3.1) we have $\sigma_{m}^{\#} \cdot \mu \boldsymbol{A}(\mathcal{A}) \cdot l_{m}=\sigma_{m}^{\#} \cdot \bigcup_{(n, \alpha) \in \mathcal{A}} \alpha \cdot r_{n}^{\#} \cdot l_{m}=\bigcup_{(n, \alpha) \in \alpha} \sigma_{m}^{\#} \cdot \alpha \cdot r_{n}^{\#} \cdot l_{m}$ $\supset \bigcup_{(n, \alpha) \in \mathcal{A}} \sigma_{m}^{\#} \cdot \alpha \cdot l_{m} \cdot r_{n}^{\#} \supset \bigcup_{(n, \alpha) \in \mathcal{A}} \alpha \cdot r_{n}^{\#}=\mu \boldsymbol{A}(\mathcal{A})$ for each $m \in M$. Therefore $\mu \boldsymbol{A}(\mathcal{A})$ is an $M$-relation.

(vii) For $\mathcal{A} \in P_{M}^{2} A$, we obtain

$$
\begin{aligned}
& (n, a) \in\left(\mu \boldsymbol{A} \cdot \rho_{m}{ }^{M^{A}}\right)(\mathcal{A})=\mu \boldsymbol{A}\left(\mathcal{A} \cdot r_{m}\right) \\
& \leftrightarrow \exists \alpha \in P_{M} \boldsymbol{A} ;(n, \alpha) \in \mathcal{A} \cdot r_{m}, \quad(e, a) \in \alpha \\
& \leftrightarrow \exists \alpha \in P_{M} \boldsymbol{A} ;(n m, \alpha) \in \mathcal{A},(e, a) \in \alpha \\
& \leftrightarrow(n m, a) \in \mu \boldsymbol{A}(\mathcal{A}) \\
& \leftrightarrow(n, a) \in \mu \boldsymbol{A}(\mathcal{A}) \cdot r_{m}=\left(\rho_{m}^{A} \cdot \mu \boldsymbol{A}\right)(\mathcal{A}) .
\end{aligned}
$$

(viii) For $A \in P_{M}^{2} A$, we obtain

$$
\begin{aligned}
& (m, b) \in\left(\mu \boldsymbol{B} \cdot P_{M}^{2} \Phi\right)(\mathcal{A})=\mu \boldsymbol{B}\left(P_{M} \Phi \cdot \mathcal{A}\right) \\
& \leftrightarrow \exists \beta \in P_{M} \boldsymbol{B} ;(m, \beta) \in P_{M} \Phi \cdot \mathcal{A},(e, b) \in \beta \\
& \leftrightarrow \exists \alpha \in P_{M} \boldsymbol{A} ;(m, \alpha) \in \mathcal{A},(e, b) \in P_{M} \Phi(\alpha)=\Phi \cdot \alpha(=\beta, \\
& \leftrightarrow \exists \alpha \in P_{M} \boldsymbol{A}, \exists a \in \boldsymbol{A} ;(m, \alpha) \in \mathcal{A},(e, a) \in \alpha, b=\Phi(a) \\
& \leftrightarrow \exists a \in \boldsymbol{A} ;(m, a) \in \mu \boldsymbol{A}(\mathcal{A}), b=\Phi(a) \\
& \leftrightarrow(m, b) \in \Phi \cdot \mu \boldsymbol{A}(\mathcal{A})=\left(P_{M} \Phi \cdot \mu \boldsymbol{A}\right)(\mathcal{A}) .
\end{aligned}
$$

We denote by $\boldsymbol{P}=\left(P, \eta^{P}, \mu^{P}\right)$ the powerset monad in Set $[3,4]$.

Theorem 3.1.

(a) $\boldsymbol{P}_{M}=\left(P_{M}, \eta_{M}^{P}, \mu_{M}^{P}\right)$ is a monad in $M$-Set.

(b) If $M=1$, then $\boldsymbol{P}_{1}$ is the powerset monad $\boldsymbol{P}$ in Set.

Proof. (a) Let $\alpha \in P_{M} A$ and $\mathscr{I} \in P_{M}^{3} A$.

(i) $\mu \boldsymbol{A} \cdot \eta P_{M} \boldsymbol{A}(\alpha)=\bigcup_{(n, \gamma) \in \eta P_{M} A(\alpha) \gamma \cdot r_{n}^{\#}}=\bigcup_{n \in M} \rho_{n}^{A}(\alpha) \cdot r_{n}^{\#}=\bigcup_{n \in M} \alpha \cdot r_{n} \cdot r_{n}^{\#}=\alpha \quad$ (by (3.1)). Therefore $\mu \cdot \eta P_{M}=\mathbf{1}_{P_{M}}$.

$$
\begin{aligned}
& (n, a) \in\left(\mu \boldsymbol{A} \cdot P_{M} \eta \boldsymbol{A}\right)(\alpha)=\mu \boldsymbol{A}(\eta \boldsymbol{A} \cdot \alpha) \\
& \leftrightarrow \exists \gamma \in P_{M} \boldsymbol{A} ;(n, \gamma) \in \eta \boldsymbol{A} \cdot \alpha,(e, a) \in \gamma \\
& \leftrightarrow \exists c \in \boldsymbol{A} ;(n, c) \in \alpha,(e, a) \in \eta \boldsymbol{A}(c)(=\gamma) \\
& \leftrightarrow(n, a) \in \alpha .
\end{aligned}
$$

Therefore $\mu \cdot P_{M} \eta=\mathbf{1}_{P_{M}}$.

(iii)

$$
\begin{aligned}
& (m, a) \in\left(\mu \boldsymbol{A} \cdot P_{M} \mu \boldsymbol{A}\right)(\mathscr{F})=\mu \boldsymbol{A}(\mu \boldsymbol{A} \cdot \mathscr{I}) \\
& \leftrightarrow \exists \alpha \in P_{M} \boldsymbol{A} ;(m, \alpha) \in \mu A \cdot \mathscr{F},(e, a) \in \alpha \\
& \leftrightarrow \exists \xi \in P_{M}^{2} \boldsymbol{A} ;(m, \xi) \in \mathscr{F},(e, a) \in \mu \boldsymbol{A}(\xi)(=\alpha) \\
& \leftrightarrow \exists \xi \in P_{M}^{2} \boldsymbol{A}, \quad \exists \gamma \in P_{M} \boldsymbol{A} ;(m, \xi) \in \mathscr{F},(e, \gamma) \in \xi,(e, a) \in \gamma \\
& \leftrightarrow \exists \gamma \in P_{M} \boldsymbol{A} ;(m, \gamma) \in \mu P_{M} \boldsymbol{A}(\mathscr{F}),(e, a) \in \gamma \\
& \leftrightarrow(m, a) \in \mu \boldsymbol{A}\left(\mu P_{M} \boldsymbol{A}(\mathscr{F})\right)=\left(\mu \boldsymbol{A} \cdot \mu P_{M} \boldsymbol{A}\right)(\mathscr{F}) .
\end{aligned}
$$

Therefore $\mu \cdot P_{M} \mu=\mu \cdot \mu P_{M}$.

(b) It is obvious that $\boldsymbol{P}_{1}$ is the powerset monad $\boldsymbol{P}$ in Set. 
Proposition 3.2. (a) For an $M$-set $\boldsymbol{A}=(A, \sigma)$ a map $s_{F}: \bar{P} \boldsymbol{A} \rightarrow P_{M} \boldsymbol{A}$, defined by $s_{P} \boldsymbol{A}(\alpha)=\bigcup_{a \in \alpha} \eta_{M} \boldsymbol{A}(a)(\alpha \in \bar{P} \boldsymbol{A})$, induces a natural transformation $s_{P}: \bar{P} \rightarrow P_{M}$.

(b) The natural transformation $s_{P}: \bar{P} \rightarrow P_{M}$ is algebraic.

Proof. (a) Let $\boldsymbol{A}=(A, \sigma)$ and $\boldsymbol{B}=(B, \tau)$ be $M$-sets and $\Phi: \boldsymbol{A} \rightarrow \boldsymbol{B}$ an $M$-morphism. Then we obtain $\left(P_{M} \Phi \cdot s \boldsymbol{A}\right)(\alpha)=\Phi \cdot s \boldsymbol{A}(\alpha)=\cup_{a \in \alpha} \Phi \cdot \eta_{M} \boldsymbol{A}(a)=\bigcup_{a \in \alpha}\left(P_{M} \Phi \cdot \eta_{M} \boldsymbol{A}\right)(a)=$ $\bigcup_{a \in \alpha}\left(\eta_{M} \boldsymbol{B} \cdot \Phi\right)(a)=\bigcup_{b \in \Phi \cdot \alpha} \eta_{M} \boldsymbol{B}(b)=(s \boldsymbol{B} \cdot \bar{P} \Phi)(\alpha)$.

For $m \in M$ and $\alpha \in \bar{P} \boldsymbol{A}$ we have $\left(\rho_{m}^{A} \cdot s \boldsymbol{A}\right)(\alpha)=\bigcup_{a \in \alpha} \eta_{M} \boldsymbol{A}(a) \cdot r_{m}=\bigcup_{a \in \alpha}\left(\rho_{m}^{A} \cdot \eta_{M} \boldsymbol{A}\right)(a)=$ $\bigcup_{a \in \alpha}\left(\eta_{M} \boldsymbol{A} \cdot \sigma_{m}\right)(a)=\bigcup_{c \in \sigma_{m} \cdot \alpha} \eta_{M} \boldsymbol{A}(c)=s \boldsymbol{A}\left(P \sigma_{m}(\alpha)\right)=\left(s \boldsymbol{A} \cdot P \sigma_{m}\right)(\alpha)$.

Therefore $s \boldsymbol{A}$ is an $M$-morphism and $s_{P}$ is a natural transformation.

(b) Next we check that $s_{P}$ is algebraic.

(i) For $a \in \boldsymbol{A}$ we have $s \boldsymbol{A} \cdot \bar{\eta} \boldsymbol{A}(a)=s \boldsymbol{A}(\{a\})=\eta_{\boldsymbol{M}} \boldsymbol{A}(a)$. Therefore $s \boldsymbol{A} \cdot \bar{\eta}_{\boldsymbol{A}}=\eta_{\boldsymbol{M}} \boldsymbol{A}$.

(ii) For $A \in \bar{P}^{2} \boldsymbol{A}$ then we have

$$
\begin{aligned}
& (m, a) \in\left(\mu_{M} \boldsymbol{A} \cdot s P_{M} \boldsymbol{A} \cdot \bar{P}_{S} \boldsymbol{A}\right)(\mathcal{A}) \\
& \leftrightarrow \exists \xi \in P_{M} \boldsymbol{A} ;(m, \xi) \in\left(s P_{\boldsymbol{M}} \boldsymbol{A} \cdot \bar{P}_{S} \boldsymbol{A}\right)(\mathcal{A}),(e, a) \in \xi \\
& \leftrightarrow \exists \xi \in P_{\boldsymbol{M}} \boldsymbol{A}, \exists \alpha \in \bar{P}_{S} \boldsymbol{A}(\mathcal{A}) ;(m, \xi) \in \eta_{M} P_{M} \boldsymbol{A}(\alpha),(e, a) \in \xi \\
& \leftrightarrow \exists \alpha \in \bar{P}_{S} \boldsymbol{A}(\mathcal{A}) ;(e, a) \in \rho_{m}^{A}(\alpha)=\alpha \cdot r_{m}(=\xi) \\
& \leftrightarrow \exists \alpha \in \bar{P}_{S} \boldsymbol{A}(\mathcal{A}) ;(m, a) \in \alpha \\
& \leftrightarrow \exists \gamma \in \mathcal{A} ;(m, a) \in s \boldsymbol{A}(\gamma)=\bigcup_{c \in \gamma} \eta_{M} \boldsymbol{A}(c)(=\alpha) \\
& \leftrightarrow \exists \gamma \in \mathcal{A}, \exists c \in \gamma ;(m, a) \in \eta_{M} \boldsymbol{A}(c) \\
& \leftrightarrow \exists c \in \bar{\mu} \boldsymbol{A}(\mathcal{A}) ;(m, a) \in \eta_{M} \boldsymbol{A}(c) \\
& \leftrightarrow(m, a) \in(s \boldsymbol{A} \cdot \bar{\mu} \boldsymbol{A})(\mathcal{A}) .
\end{aligned}
$$

Therefore $s \boldsymbol{A} \cdot \bar{\mu} \boldsymbol{A}=\mu_{M} \boldsymbol{A} \cdot s P_{M} \boldsymbol{A} \cdot \bar{P} s \boldsymbol{A}$.

Since the condition (2.1) holds, then $s_{P}$ is algebraic.

For an $M$-set $\boldsymbol{A}$ we define a map $t_{P} \boldsymbol{A}: P_{M} \boldsymbol{A} \rightarrow \bar{P} \boldsymbol{A}$ by $t_{P} \boldsymbol{A}(\alpha):=\alpha \cdot e\left(\alpha \in P_{M} \boldsymbol{A}\right)$. We note that the unit element $e \in M$ is identified with a map $e: 1 \rightarrow M$.

Proposition 3.3. $t_{P}$ is a natural transformation iff $M$ is a group.

Proof. First we obtain $\bar{P} \Phi \cdot t \boldsymbol{A}=t \boldsymbol{B} \cdot P_{M} \Phi$ because $(\bar{P} \Phi \cdot t \boldsymbol{A})(\alpha)=\bar{P} \Phi(\alpha \cdot e)=\Phi \cdot \alpha \cdot e=$ $\left({ }_{t} \boldsymbol{B} \cdot P_{M} \Phi\right)(\alpha)$ for $\alpha \in P_{M} \boldsymbol{A}$. Next we have $\left(P \sigma_{m} \cdot t \boldsymbol{A}\right)(\alpha)=P \sigma_{m}(\alpha \cdot e)=\sigma_{m} \cdot \alpha \cdot e$ and $\left(t \boldsymbol{A} \cdot \rho_{m}^{A}\right)(\alpha)$ $=t \boldsymbol{A}\left(\alpha \cdot r_{m}\right)=\alpha \cdot r_{m} \cdot e$ for each $m \in M$. The relation $\sigma_{m} \cdot \alpha \cdot e \subset \alpha \cdot r_{m} \cdot e(m \in M)$ is trivial. To show that $t_{P}$ is a natural transformation, we only show $\sigma_{m} \cdot \alpha \cdot e \supset \alpha \cdot r_{m} \cdot e(m \in M)$.

If $M$ is a group, then $a \in \alpha \cdot r_{m} \cdot e$ implies $(m, a) \in \alpha,\left(e, \sigma_{m-1}(a)\right) \in \alpha$ and $a \in \sigma_{m} \cdot \alpha \cdot e$. So we have $\sigma_{m} \cdot \alpha \cdot e=\alpha \cdot r_{m} \cdot e$. Therefore $t_{P}$ is a natural transformation.

Conversely, assume that $t_{P}$ is natural. Then we have $\alpha \cdot r_{m} \cdot e \subset \sigma_{m} \cdot \alpha \cdot e$ for any $M$-set $\boldsymbol{A}, a \in \boldsymbol{A}$ and $m \in M$. If we assign $\boldsymbol{A}:=(M, l)$ and $\alpha:=M \times M$, then there exists $n \in \alpha \cdot e$ such that $l_{m}(n)=m n=e$ for any $m \in M$ since $e \in \alpha \cdot r_{m} e \subset l_{m} \cdot \alpha \cdot e$. This shows that $M$ is a group.

THEOREM 3.4. If $M$ is a group, then the algebraic functor $s_{P}^{*}: M-$ Set $^{P} M \rightarrow M-$ Set $^{\bar{P}}$ is an isomorphism of categories.

Proof. By Lemma 2.5, we must only show that $s$ is an isomorphism. Let $\boldsymbol{A}$ be an $M$-set and $\alpha \in \bar{P} \boldsymbol{A}$. Then we have $(t \boldsymbol{A} \cdot s \boldsymbol{A})(\alpha)=s \boldsymbol{A}(\alpha) \cdot e=\bigcup_{a \in \alpha} \eta_{\boldsymbol{M}} \boldsymbol{A}(a) \cdot e=\alpha$. Therefore $t \boldsymbol{A} \cdot s \boldsymbol{A}=\mathbf{1}_{\bar{P} A}$.

Next we note that $(s \boldsymbol{A} \cdot t \boldsymbol{A})(\alpha)=\bigcup_{a \in \alpha \cdot e} \eta_{M} \boldsymbol{A}(a)=\bigcup_{(e, a) \in \alpha} \eta_{M} \boldsymbol{A}(a)$. It is obvious that $\bigcup_{(e, a) \in \alpha} \eta_{M} \boldsymbol{A}(a) \subset \alpha$, and, since $M$ is a group, $(m, b) \in \alpha$ implies $\left(l_{m-1}(m), \sigma_{m^{-1}}(b)\right)=$ 
$\left(e, \sigma_{m-1}(b)\right) \equiv \alpha$ and $(m, b) \in \eta_{M} \boldsymbol{A}\left(\sigma_{m-1}(b)\right)$. So we have $\bigcup_{(e, a) \in \alpha} \eta_{M} \boldsymbol{A}(a)=\alpha$ and $s \boldsymbol{A} \cdot \boldsymbol{t} \boldsymbol{A}$ $=\mathbf{1}_{P_{M} A}$.

We denote by CSL, the category of complete semilattices and arbitrary supremum preserving maps. It is known that $\mathrm{CSL} \cong \operatorname{Set}^{P}$ as categories. So we obtain the next corollary by the last theorem and Proposition 2.4 .

COROLLARY 3.5. If $M$ is a group, then

$$
M-\operatorname{Set}^{P} M \cong M-\left(\operatorname{Set}^{P}\right) \cong M-\mathrm{CSL} .
$$

By this corollary, if $M$ is a group then a $\boldsymbol{P}_{M}$-algebra is identified with the a complete semilattice with a supremum preserving $M$-action.

\section{Filter Monad in M-Set}

In this section, we define a filter on $M$-sets by using the order (inclusion) of $M$-relations, a filter monad $F_{M}$ in $M$-Set and an algebraic functor $s_{F}^{*}: M$-Set ${ }^{F_{M}} \rightarrow M$-Set ${ }^{\bar{F}}$. Moreover we show that $s_{F}^{*}$ is an isomorphism if $M$ is a group.

A filter on an $M$-set $\boldsymbol{A}$ is a subset $f \subset \Omega^{A}$ that satisfies

(F1) $\Theta \in f \quad($ where $\Theta:=M \times A$ ),

(F2) if $\alpha, \beta \in f$ then $\alpha \cap \beta \in f$,

(F3) if $\alpha \in f$ and $\alpha \subset \beta\left(\in \Omega^{A}\right)$ then $\beta \in f$.

We note that if $\alpha, \beta \in \Omega^{A}$ then $\alpha \cap \beta \in \Omega^{A}$.

To obtain an endofunctor $F_{M}: M$-Set $\rightarrow M$-Set, we define $F_{M} \boldsymbol{A}$ to be the set of all filters on $\boldsymbol{A}$, and the $M$-action $\zeta^{A}$ on $F_{M} \boldsymbol{A}$ by

for $f \in F_{M} \boldsymbol{A}$ and $m \in M$.

$$
\alpha \in \zeta_{m}^{A}(f) \quad \text { iff } \quad \sigma_{m}^{\sharp} \cdot \alpha \in f
$$

For an $M$-morphism $\Phi: \boldsymbol{A} \rightarrow \boldsymbol{B}$ we define $F_{M} \Phi: F_{M} \boldsymbol{A} \rightarrow F_{M} \boldsymbol{B}$ by

for $f \in F_{M} A$.

$$
\beta \in F_{M} \Phi(f) \quad \text { iff } \quad \Phi^{\#} \cdot \beta \in f
$$

To check that $F_{M}$ is a functor, we verify the followings.

(i) $\zeta_{m}(f)$ is a filter on $\boldsymbol{A}$ for $m \in M$.

Since $\sigma_{m}^{\#} \cdot \Theta=\Theta \in f$ we obtain $\Theta \in \zeta_{m}(f)$. If $\alpha, \beta \in \zeta_{m}(f)$ then we have $\sigma_{m}^{\#} \cdot(\alpha \cap \beta)=$ $\left(\sigma_{m}^{\#} \cdot \alpha\right) \cap\left(\sigma_{m}^{\#} \cdot \beta\right) \in f$ and so $\alpha \cap \beta \in \zeta_{m}(f)$. If $\alpha \in \zeta_{m}(f)$ and $\alpha \subset \beta \in \Omega^{A}$ then we have $\beta \in \zeta_{m}(f)$ by $\left(\sigma_{m}^{\#} \cdot \alpha\right) \subset\left(\sigma_{m}^{\#} \cdot \beta\right) \in f$.

(ii) $F \Phi(f)$ is a filter on $\boldsymbol{B}$.

It is similar to (i).

(iii) $\zeta_{m}^{B} \cdot F \Phi=F \Phi \cdot \zeta_{m}^{A}$ for $m \in M$.

For $f \in F_{M} A$ we have $\beta \in\left(F \Phi \cdot \zeta_{m}^{A}\right)(f) \leftrightarrow \Phi^{\#} \cdot \beta \in \zeta_{m}^{A}(f) \leftrightarrow \sigma_{m}^{\#} \cdot \Phi^{\#} \cdot \beta \in f \leftrightarrow \Phi^{\#} \cdot \tau_{m}^{\#} \cdot \beta \in f \leftrightarrow$ $\tau_{m}^{\#} \cdot \beta \in F \Phi(f) \leftrightarrow \beta \in\left(\zeta_{m}^{B} \cdot F \Phi\right)(f)$.

Next three maps $\eta_{m}^{F} \boldsymbol{A}: \boldsymbol{A} \rightarrow F_{M} \boldsymbol{A}, \pi_{M}^{F} A: \Omega^{\Lambda} \rightarrow \Omega^{F_{M}}{ }^{A}$ and $\mu_{M}^{F} \boldsymbol{A}: F_{M}^{2} \boldsymbol{A} \rightarrow F_{M} \boldsymbol{A}$ for an $M$-set $\boldsymbol{A}$ are defined by 


$$
\begin{array}{lll}
\alpha \in \eta_{M}^{F} \boldsymbol{A}(a) & \text { iff } & (e, a) \equiv \alpha, \\
(m, f) \in \pi_{M}^{F} \boldsymbol{A}(\alpha) & \text { iff } & \alpha \cdot l_{m} \in f, \\
\alpha \in \mu_{M}^{F} \boldsymbol{A}(\mathscr{I}) & \text { iff } & \pi_{M}^{F} \boldsymbol{A}(\alpha) \subseteq \mathscr{I}
\end{array}
$$

for $a \in A, \alpha \in Q^{A}$ and $\mathscr{F} \in F_{M}^{2} A$.

For $M$-sets $\boldsymbol{A}=(A, \sigma), \boldsymbol{B}=(B, \tau)$ and an $M$-morphism $\Phi: \boldsymbol{A} \rightarrow \boldsymbol{B}$ the following facts holds :

(i) $\eta_{M}^{F} \boldsymbol{A}(a)$ is a filter on $\boldsymbol{A}$ for $a \in \boldsymbol{A}$.

(ii) $\zeta_{m}^{A} \cdot \eta_{M}^{F} \boldsymbol{A}=\eta_{M}^{F} \boldsymbol{A} \cdot \sigma_{m}$ for $m \in M$.

For $a \in \boldsymbol{A}$ we have $\alpha \in\left(\zeta_{m}^{A} \cdot \eta \boldsymbol{A}\right)(a) \leftrightarrow \sigma_{m}^{\#} \cdot \alpha \equiv \gamma_{1} \boldsymbol{A}(a) \leftrightarrow(e, a) \Xi \sigma_{m}^{\#} \cdot \alpha \leftrightarrow\left(e, \sigma_{m}(a)\right) \equiv \alpha \leftrightarrow$ $\alpha \in \eta \boldsymbol{A}\left(\sigma_{m}(a)\right) \leftrightarrow \alpha \in\left(\eta \boldsymbol{A} \cdot \sigma_{m}\right)(a)$.

(iii) $F_{M} \Phi \cdot \eta_{M}^{F} A=\eta_{M}^{F} B \cdot \Phi$.

For $a \in \boldsymbol{A}$ we have $\alpha \in\left(F_{M} \Phi \cdot \eta \boldsymbol{A}\right)(a) \leftrightarrow \Phi^{\ddagger} \cdot \alpha \in \gamma_{1} \boldsymbol{A}(a) \leftrightarrow(e, a) \in \Phi^{*} \cdot \alpha \leftrightarrow(e, \Phi(a)) \subseteq \alpha \leftrightarrow$ $\alpha \in \eta \boldsymbol{B}(\Phi(a)) \leftrightarrow \alpha \in(\eta \boldsymbol{B} \cdot \Phi)(a)$.

(iv) $\mu_{M}^{F} \boldsymbol{A}(\mathscr{F})$ is a filter for $\mathscr{F} \in F_{M}^{2} \boldsymbol{A}$.

Since $\pi \boldsymbol{A}(\Theta)=M \times F_{M} \boldsymbol{A} \in \mathscr{I}$ we have $\Theta \in \mu \boldsymbol{A}(\mathscr{F})$. If $\alpha, \beta \in \mu \boldsymbol{A}(\mathscr{F})$ then we have $\pi \boldsymbol{A}(\alpha), \pi \boldsymbol{A}(\beta) \in \mathscr{I}$, so $(m, f) \in \pi \boldsymbol{A}(\alpha \cap \beta) \leftrightarrow(\alpha \cap \beta) \cdot l_{m} \in f \leftrightarrow \alpha \cdot l_{m} \cap \beta \cdot l_{m} \subseteq f \leftrightarrow \alpha \cdot l_{m} \in f$ and $\beta \cdot l_{m} \in f \leftrightarrow(m, f) \in \boldsymbol{\pi} \boldsymbol{A}(\alpha) \cap \boldsymbol{\pi} \boldsymbol{A}(\beta)$. Therefore $\pi \boldsymbol{A}(\alpha \cap \beta)=\pi \boldsymbol{A}(\alpha) \cap \boldsymbol{\pi} \boldsymbol{A}(\beta) \in \mathscr{I}$. If $\alpha \in \mu \boldsymbol{A}(\mathscr{I})$ and $\alpha \subset \beta$ then we have $\pi \boldsymbol{A}(\alpha) \in \mathscr{F}$, so $(m, f) \in \pi \boldsymbol{A}(\alpha) \leftrightarrow \alpha \cdot l_{m} \subseteq f \rightarrow \beta \cdot l_{m} \in f \leftrightarrow(m, f) \in \pi \boldsymbol{A}(\beta)$. Therefore $\pi \boldsymbol{A}(\alpha) \subset \pi \boldsymbol{A}(\beta) \in \mathscr{F}$.

(v) $\mu_{M}^{F} \boldsymbol{A} \cdot \zeta_{m}{ }^{F}{ }_{M}{ }^{A}=\zeta_{m}^{A} \cdot \mu_{M}^{F} \boldsymbol{A}$ for $m \in M$.

For $\mathscr{I} \in F_{M}^{2} \boldsymbol{A}$ we obtain $\alpha \in\left(\mu \boldsymbol{A} \cdot \zeta_{m} F_{M}^{A}\right)(\mathscr{I}) \leftrightarrow \pi \boldsymbol{A}(\alpha) \in \zeta_{m} F_{M}^{A}(\mathscr{I}) \leftrightarrow\left(\zeta_{m}^{A}\right)^{\#} \cdot \pi \boldsymbol{A}(\alpha) \subseteq \mathscr{I} \leftrightarrow$ $\pi \boldsymbol{A}\left(\sigma_{m}^{\#} \cdot \alpha\right) \in \mathscr{T} \leftrightarrow \sigma_{m}^{\#} \cdot \alpha \in \mu \boldsymbol{A}(\mathscr{F}) \leftrightarrow \alpha \in\left(\zeta_{m}^{A} \cdot \mu \boldsymbol{A}\right)(\mathscr{F})$, since $(n, f) \in \pi \boldsymbol{A}\left(\sigma_{m}^{\sharp} \cdot \alpha\right) \leftrightarrow \sigma_{m}^{\sharp} \cdot \alpha \cdot l_{n} \in f \leftrightarrow$ $\alpha \cdot l_{n} \in \zeta_{m}^{A}(f) \leftrightarrow\left(n, \zeta_{m}^{A}(f)\right) \in \pi A(\alpha) \leftrightarrow(n, f) \in\left(\zeta_{m}^{A}\right)^{\#} \cdot \pi \boldsymbol{A}(\alpha)$.

(vi) $\mu_{M}^{F} B \cdot F_{M}^{2} \Phi=F_{M} \Phi \cdot \mu_{M}^{F} A$.

For $\mathscr{T} \in F_{M}^{2} \boldsymbol{A}$ we have $\alpha \in\left(\mu \boldsymbol{B} \cdot F_{M}^{2} \Phi\right)(\mathscr{T}) \leftrightarrow \pi \boldsymbol{B}(\alpha) \in F_{M}^{2} \Phi(\mathscr{I}) \leftrightarrow\left(F_{M} \Phi\right)^{\ddagger} \cdot \pi \boldsymbol{B}(\alpha) \in \mathscr{I} \leftrightarrow$ $\pi \boldsymbol{A}\left(\Phi^{\#} \cdot \alpha\right) \in \mathscr{I} \leftrightarrow \Phi^{\#} \cdot \alpha \in \mu \boldsymbol{A}(\mathscr{I}) \leftrightarrow \alpha \in\left(F_{M} \Phi \cdot \mu \boldsymbol{A}\right)(\mathscr{I}), \quad$ since $\quad(m, f) \in\left(F_{M} \Phi\right)^{\sharp} \cdot \pi \boldsymbol{B}(\alpha) \leftrightarrow$ $\left(m, F_{M} \Phi(f)\right) \in \pi \boldsymbol{B}(\alpha) \leftrightarrow \alpha \cdot l_{m} \in F_{M} \Phi(f) \leftrightarrow \Phi^{\#} \cdot \alpha \cdot l_{m} \in f \leftrightarrow(m, f) \in \pi \boldsymbol{A}\left(\Phi^{\sharp} \cdot \alpha\right)$.

The facts (i)-(vi) show that $\eta_{M}^{F}: 1_{M \text {-set }} \rightarrow F_{M}$ and $\mu_{M}^{F}: F_{M}^{2} \rightarrow F_{M}$ are natural transformations.

Proposition 4.1. $\pi_{M}^{F} \boldsymbol{A}$ is an $M$-morphism for any $M$-set $\boldsymbol{A}$ iff $M$ is abelian.

PROOF. For any $n \in M$ we have $(m, f) \in\left(\rho_{n}{ }^{F_{M}} \cdot \pi \boldsymbol{A}\right)(\alpha) \leftrightarrow(m, f) \in \pi \boldsymbol{A}(\alpha) \cdot r_{n} \leftrightarrow$ $(m n, f) \in \pi \boldsymbol{A}(\alpha) \leftrightarrow \alpha \cdot l_{m n} \in f \leftrightarrow \alpha \cdot l_{m} \cdot l_{n} \in f$ and $(m, f) \in\left(\pi \boldsymbol{A} \cdot \boldsymbol{\rho}_{n}^{A}\right)(\alpha) \leftrightarrow(m, f) \in \pi \boldsymbol{A}\left(\alpha \cdot r_{n}\right) \leftrightarrow$ $\alpha \cdot \gamma_{n} \cdot l_{m} \in f \leftrightarrow \alpha \cdot l_{m} \cdot \gamma_{n} \in f$. So we can reduce the condition $\rho_{n}{ }^{F_{M}}{ }^{A} \cdot \pi \boldsymbol{A}=\pi \boldsymbol{A} \cdot \rho_{n}^{A}(n \in M)$ to

$$
\alpha \cdot l_{m} \cdot l_{n}=\alpha \cdot l_{m} \cdot r_{n}\left(\alpha \in \Omega^{A}, n, m \in M\right) .
$$

If $M$ is abelian then the equation (4.1) is trivial. Conversely, if the condition (4.1) is valid then by assigning $\boldsymbol{A}=\boldsymbol{M}, \alpha=\mathbf{1}_{M}$ and $m=e$ we have $\mathbf{1}_{M} \cdot l_{e} \cdot l_{n}(k)=\mathbf{1}_{M} \cdot l_{e} \cdot r_{n}(k)$ for any $n, k \in M$. This means $n k=k n$ for any $n, k \in M$. Therefore $M$ is abelian.

LEMMA 4.2. For each $\alpha \in \Omega^{A}$,

(a) $\mu_{M}^{F} A^{\#} \cdot \pi_{M}^{F} \boldsymbol{A}(\alpha)=\left(\pi_{M}^{F} F_{M} A \cdot \pi_{M}^{F} \boldsymbol{A}\right)(\alpha)$,

(b) $\eta_{M}^{F} A^{\#} \cdot \pi_{M}^{F} A(\alpha)=\alpha$.

Proof. (a) Since $(n, f) \in \pi \boldsymbol{A}(\alpha) \cdot l_{m} \leftrightarrow(m n, f) \equiv \pi \boldsymbol{A}(\alpha) \leftrightarrow \alpha \cdot l_{m n} \in f \leftrightarrow \alpha \cdot l_{m} \cdot l_{n} \in f \leftrightarrow$ $(n, f) \in \pi \boldsymbol{A}\left(\alpha \cdot l_{m}\right)$, we obtain $(m, \mathscr{F}) \in\left(\pi F_{M} \boldsymbol{A} \cdot \pi \boldsymbol{A}\right)(\alpha) \leftrightarrow \pi \boldsymbol{A}(\alpha) \cdot l_{m} \in \mathscr{I} \leftrightarrow \pi \boldsymbol{A}\left(\alpha \cdot l_{m}\right) \in \mathscr{I} \leftrightarrow$ 
$\alpha \cdot l_{m} \in \mu \boldsymbol{A}(\mathscr{F}) \leftrightarrow(m, \mu \boldsymbol{A}(\mathscr{F})) \in \pi \boldsymbol{A}(\alpha) \leftrightarrow(m, \mathscr{F}) \in \mu \boldsymbol{A}^{\mp} \cdot \pi \boldsymbol{A}(\alpha)$.

(b) $(m, a) \in \eta \boldsymbol{A}^{\#} \cdot \pi \boldsymbol{A}(\alpha) \leftrightarrow(m, \eta \boldsymbol{A}(a)) \in \pi \boldsymbol{A}(\alpha) \leftrightarrow \alpha \cdot l_{m} \in \eta \boldsymbol{A}(a) \leftrightarrow(e, a) \in \alpha \cdot l_{m} \leftrightarrow(m, a) \equiv \alpha$. We denote by $\boldsymbol{F}=\left(F, \eta^{F}, \mu^{F}\right)$ the filter monad in Set [1].

THEOREM 4.3 .

(a) $\boldsymbol{F}_{M}=\left(F_{M}, \eta_{M}^{F}, \mu_{M}^{F}\right)$ is a monad in $M$-Set.

(b) If $M=1$, then $\boldsymbol{F}_{1}$ is the usual filter monad $\boldsymbol{F}$ in Set.

Proof. (a) Let $f \in F_{M} A$ and $\mathscr{F} \in F_{M}^{3} A$.

(i) $\quad \alpha \in\left(\mu \boldsymbol{A} \cdot \eta F_{M} \boldsymbol{A}\right)(f) \leftrightarrow \pi \boldsymbol{A}(\alpha) \in \eta F_{M} \boldsymbol{A}(f) \leftrightarrow(e, f) \in \pi \boldsymbol{A}(\alpha) \leftrightarrow \alpha \cdot l_{e} \in f \leftrightarrow \alpha \in f$. Therefore $\mu \cdot \eta F_{M}=1_{F_{M}}$.

(ii) $\alpha \in\left(\boldsymbol{\mu} \boldsymbol{A} \cdot F_{M} \eta \boldsymbol{A}\right)(f) \leftrightarrow \pi \boldsymbol{A}(\alpha) \in F_{M} \eta \boldsymbol{A}(f) \leftrightarrow \eta \boldsymbol{A}^{\#} \cdot \pi \boldsymbol{A}(\alpha) \in f \leftrightarrow \alpha \in f$ by Lemma 4.2 (b). Therefore $\mu \cdot F_{M} \eta=\mathbf{1}_{F_{M}}$.

(iii) $\quad \alpha \in\left(\mu \boldsymbol{A} \cdot \mu F_{M} \boldsymbol{A}\right)(\mathscr{I}) \leftrightarrow \pi \boldsymbol{A}(\alpha) \in \mu F_{M} \boldsymbol{A}(\mathscr{F}) \leftrightarrow\left(\pi F_{M} \boldsymbol{A} \cdot \pi \boldsymbol{A}\right)(\alpha) \in \mathscr{F} \leftrightarrow \mu \boldsymbol{A}^{\#} \cdot \pi \boldsymbol{A}(\alpha) \in \mathscr{F} \leftrightarrow$ $\pi \boldsymbol{A}(\alpha) \in F_{M} \mu \boldsymbol{A}(\mathscr{I}) \leftrightarrow \alpha \in\left(\mu \boldsymbol{A} \cdot F_{M} \mu \boldsymbol{A}\right)(\mathscr{F})$ by Lemma $4.2(\mathrm{a})$. Therefore $\mu \cdot \mu F_{M}=\mu \cdot F_{M} \mu$.

(b) It is obvious to see that $\boldsymbol{F}_{1}$ is the filter monad $\boldsymbol{F}$ in Set.

We note that $\alpha \in \mu A(\mathscr{F})$ iff $\pi F_{1} A(\alpha) \in \mathscr{F}$ for a set $A$ and $\mathscr{I} \in F^{2} A$.

PROPOSITION 4.4 .

(a) For an $M$-set $\boldsymbol{A}$ a map $s_{F} \boldsymbol{A}: \bar{F} \boldsymbol{A} \rightarrow F_{M} \boldsymbol{A}$, defined by

$$
\alpha \in s_{F} \boldsymbol{A}(f) \quad \text { iff } \quad \alpha \cdot e \in f \quad(f \in \bar{F} \boldsymbol{A}),
$$

induces a natural transformation $s_{F}: \bar{F} \rightarrow F_{M}$.

(b) The natural transformation $s_{F}: \bar{F} \rightarrow F_{M}$ is algebraic.

Proof. (a) Let $\boldsymbol{A}=(A, \sigma)$ and $\boldsymbol{B}=(B, \tau)$ are $M$-sets, $\Phi: \boldsymbol{A} \rightarrow \boldsymbol{B}$ an $M$-morphisms and $m \in M$. Then it follows that $\beta \in\left(F_{M} \Phi \cdot s \boldsymbol{A}\right)(f) \leftrightarrow \Phi^{\#} \cdot \beta \in s \boldsymbol{A}(f) \leftrightarrow \Phi^{\#} \cdot \beta \cdot e \in f \leftrightarrow$ $\beta \cdot e \in \bar{F} \Phi(f) \leftrightarrow \beta \in(s \boldsymbol{B} \cdot \bar{F} \Phi)(f), \quad$ and $\quad \alpha \in\left(\zeta_{m}^{A} \cdot s \boldsymbol{A}\right)(f) \leftrightarrow \sigma_{m}^{\#} \cdot \alpha \in s \boldsymbol{A}(f) \leftrightarrow \sigma_{m}^{\#} \cdot \alpha \cdot e \in f \leftrightarrow$ $\alpha \cdot e \in F \sigma_{m}(f) \leftrightarrow \alpha \in\left(s \boldsymbol{A} \cdot F \sigma_{m}\right)(f)$. These show that $s_{F}$ is a natural transformation.

(b) Next we check that $s_{F}$ is algebraic.

(i) For $a \in \boldsymbol{A}$, we have $\alpha \in(s \boldsymbol{A} \cdot \bar{\eta} \boldsymbol{A})(a) \leftrightarrow \alpha \cdot e \in \bar{\eta} \boldsymbol{A}(a) \leftrightarrow a \in \alpha \cdot e \leftrightarrow(e, a) \in \alpha \leftrightarrow \alpha \in \eta_{M} \boldsymbol{A}(a)$. Therefore $s \boldsymbol{A} \cdot \bar{\eta} A=\eta_{M} \boldsymbol{A}$.

(ii) For $\alpha \in F_{M} \boldsymbol{A}$ and $\mathscr{I} \in \bar{F}^{2} \boldsymbol{A}$, since $f \in(s \boldsymbol{A})^{\#} \cdot \pi_{M} \boldsymbol{A}(\alpha) \cdot e \leftrightarrow s \boldsymbol{A}(f) \in \pi_{M} \boldsymbol{A}(\alpha) \cdot e \leftrightarrow$ $(e, s \boldsymbol{A}(f)) \in \pi_{M} \boldsymbol{A}(\alpha) \leftrightarrow \alpha \in s \boldsymbol{A}(f) \leftrightarrow \alpha \cdot e \in f \leftrightarrow f \in \pi_{1} \boldsymbol{A}(\alpha \cdot e)$, we have $\alpha \in(s \boldsymbol{A} \cdot \bar{\mu} \boldsymbol{A})(\mathscr{T}) \leftrightarrow$ $\alpha \cdot e \in \bar{\mu} \boldsymbol{A}(\mathscr{F}) \leftrightarrow \pi_{1} \boldsymbol{A}(\alpha \cdot e) \in \mathscr{F} \leftrightarrow(s \boldsymbol{A})^{\#} \cdot \pi_{M} \boldsymbol{A}(\alpha) \cdot e \in \mathscr{F} \leftrightarrow \pi_{M} \boldsymbol{A}(\alpha) \cdot e \in \bar{F} S \boldsymbol{A}(\mathscr{F}) \leftrightarrow \pi_{M} \boldsymbol{A}(\alpha) \in$ $\left(s F_{M} \boldsymbol{A} \cdot \bar{F} s \boldsymbol{A}\right)(\mathscr{F}) \leftrightarrow \alpha \in\left(\mu_{M} \boldsymbol{A} \cdot s F_{M} \boldsymbol{A} \cdot \bar{F} s \boldsymbol{A}\right)(\mathscr{T})$. Therefore $s \boldsymbol{A} \cdot \bar{\mu} \boldsymbol{A}=\mu_{M} \boldsymbol{A} \cdot s F_{M} \boldsymbol{A} \cdot \bar{F} s \boldsymbol{A}$.

Hence $s_{F}$ is algebraic.

For an $M$-Set $\boldsymbol{A}$ we define a map $t_{F} \boldsymbol{A}: F_{M} \boldsymbol{A} \rightarrow \bar{F} \boldsymbol{A}$ by

$$
\alpha \in t_{F} \boldsymbol{A}(f) \quad \text { iff } \bigcup_{a \in \alpha} \eta_{\boldsymbol{M}}^{P} \boldsymbol{A}(a) \in f \quad\left(f \in F_{M} \boldsymbol{A}\right) .
$$

LEMmA 4.5. The naturality $\bar{F} \Phi \cdot t_{F} \boldsymbol{A}=t_{F} \boldsymbol{B} \cdot F_{M} \Phi$ in Set holds for any $M$-sets $\boldsymbol{A}=$ $(A, \sigma), \boldsymbol{B}=(B, \tau)$ and an $M$-morphism $\Phi: \boldsymbol{A} \rightarrow \boldsymbol{B}$ iff $M$ is a group.

Proof. For $f \in F_{\boldsymbol{M}} \boldsymbol{A}$ it follows that $\beta \in(\bar{F} \Phi \cdot t \boldsymbol{A})(f) \leftrightarrow \Phi^{\#} \cdot \beta \in t \boldsymbol{A}(f) \leftrightarrow \cup_{a \in \Phi^{\sharp}, \beta} \eta_{M}^{P} \boldsymbol{A}(a)$ $\in f$ and $\beta \in\left(t \boldsymbol{B} \cdot F_{M} \Phi\right)(f) \leftrightarrow \bigcup_{b \in \beta} \eta_{M}^{P} \boldsymbol{B}(b) \in F_{M} \Phi(f) \leftrightarrow \Phi^{\#} \cdot \bigcup_{b \in \beta} \eta_{M}^{P} \boldsymbol{B}(b) \in f$.

So the condition $\bar{F} \Phi \cdot t \boldsymbol{A}=t \boldsymbol{B} \cdot F_{M} \Phi$ for $t_{F}$ is reduced to $\bigcup_{a \in \Phi^{\sharp} \cdot \beta} \eta_{M}^{P} \boldsymbol{A}(a)=$ $\Phi^{\#} \cdot\left(\bigcup_{b \in \beta} \eta_{M}^{P} \boldsymbol{B}(b)\right)(\beta \in P B)$. Because we can easily show that the left-hand side of the last equality is a subset of the right-hand side, the naturality of $t_{F}$ is equivalent to the condition 


$$
\bigcup_{a \in \Phi^{\ddagger} \cdot \beta} \eta_{M}^{P} \boldsymbol{A}(a) \supset \Phi^{\ddagger} \cdot\left(\bigcup_{b \in \beta} \eta_{M}^{P} \boldsymbol{B}(b)\right) \quad(\beta \in P B) .
$$

Let $M$ be a group and $(m, c) \in \Phi^{\sharp} \cdot\left(\bigcup_{b \in \beta} \eta_{M}^{P} \boldsymbol{B}(b)\right)$. Then $\Phi(c)=\tau_{m}(b)$ for some $b \in \beta$. By assigning $a:=\sigma_{m-1}(c)$ we have $\Phi(a)=\left(\Phi \cdot \sigma_{m-1}\right)(c)=\left(\tau_{m-1} \cdot \Phi\right)(c)=b \in \beta$ and $\sigma_{m}(a)=c$. This shows $(m, c) \in \bigcup_{a \in \Phi^{\sharp} \cdot \beta} \eta_{M}^{P} \boldsymbol{A}(a)$.

Conversely, we assume that (4.6) is valid and put $\boldsymbol{A}=\boldsymbol{B}=\boldsymbol{M}, \Phi=r_{m}$. Then we obtain $(m, e) \in \bigcup_{a \in r_{m}^{\sharp} \cdot(e)} \eta_{M}^{P} \boldsymbol{A}(a)$, since $(m, e) \in r_{m}^{\sharp} \cdot \eta_{M}^{P} \boldsymbol{B}(e)$ for any $m \in M$. This means that there exists $a \in A$ such that $r_{m}(a)=e$ and $l_{m}(a)=e$. That is, $a \in \boldsymbol{A}=\boldsymbol{M}$ is an inverse element of $m$. Therefore $M$ is a group.

THEOREM 4.6. If $M$ is a group, then the algebraic functor $s_{F}^{*}: M-\operatorname{Set}^{F_{M}} \rightarrow M-\operatorname{Set}^{\bar{F}}$ is an isomorphism of categories.

Proof. Let $\boldsymbol{A}$ be an $M$-set. We first show that $s_{F} \boldsymbol{A}$ is an isomorphism with $t_{F} \boldsymbol{A}$ as the inverse morphism in Set. By definitions, it follows that

$$
\begin{array}{ll}
\alpha \in s_{F} \boldsymbol{A}(f) \leftrightarrow t_{P} \boldsymbol{A}(\alpha) \in f & (f \in \bar{F} \boldsymbol{A}), \\
\alpha \in t_{F} \boldsymbol{A}(f) \leftrightarrow s_{P} \boldsymbol{A}(\alpha) \in f & \left(f \in F_{M} \boldsymbol{A}\right) .
\end{array}
$$

If $M$ is a group then $t_{P} \boldsymbol{A}$ and $s_{P} \boldsymbol{A}$ are isomorphisms, so we have $s_{F} \boldsymbol{A}$ and $t_{F} \boldsymbol{A}$ are isomorphisms in Set. On the other hand, since $s_{F} \boldsymbol{A}$ is an $M$-morphism, the inverse map $t_{F} \boldsymbol{A}$ of $s_{F} \boldsymbol{A}$ is also an $M$-morphism. By Lemma 4.5, we see that $t_{F}$ is the inverse natural transformation of $s_{F}$ and that $s_{F}^{*}$ is an isomorphism of categories by Lemma 2.5 .

We denote by $\mathrm{CL}$ the category of continuous lattices and non-empty directed join (sup) and arbitrary meet(inf) preserving maps. It is known that $\mathrm{CL} \cong \operatorname{Set}^{F}$ as categories [1]. So we obtain the next corollary by the last theorem and Proposition 2.4.

COROLLARY 4.7. If $M$ is a group, then

$$
M-\operatorname{Set}^{F_{M}} \cong M-\left(\operatorname{Set}^{F}\right) \cong M-\mathrm{CL} .
$$

If $M$ is a group then, by this corollary, we can identify an $F_{M}$-algebra with a continuous lattice equipped with a non-empty directed join and arbitrary meet preserving $M$-action.

\section{Primefilter Monad in $M$-Set}

In this section, we modify the definitions of the filter monad in $\S 4$, to define a primefilter monad $U_{M}$ in $M$-Set. We show that the whole properties of the filter monad in $\S 4$ also hold for the primefilter monad.

A primefilter on an $M$-set $\boldsymbol{A}$ is a subset $f \subset \Omega^{A}$ that satisfies

(PF1) $f$ is a filter on $A$,

(PF2) $\phi \notin f$,

(PF3) if $\alpha \cup \beta \in f$ for $\alpha, \beta \in \Omega^{A}$ then $\alpha \in f$ or $\beta \in f$.

We note that if $M$ is a group then a primefilter is an ultrafilter (maximal filter).

Now by simulating the definition of the filter monad in $\S 4$, we can define a primefilter monad in $M$-Set. For example, we define an endofunctor $U_{M}: M$-Set $\rightarrow M$-Set. For an $M$-set $\boldsymbol{A}, U_{M} \boldsymbol{A}$ is the set of all primefilters on $\boldsymbol{A}$, and the $M$-action $\zeta^{A}$ on $U_{M} \boldsymbol{A}$ is defined by 


$$
\alpha \in \zeta_{m}^{A}(f) \quad \text { iff } \quad \sigma_{m}^{\sharp} \cdot \alpha \in f
$$

for $m \in M$ and $f \in U_{M} \boldsymbol{A}$. We can also define $U_{M} \Phi: U_{M} \boldsymbol{A} \rightarrow U_{M} \boldsymbol{B}, \quad \eta_{M}^{U}: \mathbf{1}_{M \text {-set }} \rightarrow U_{M}$, $\pi_{M}^{U} A: \Omega^{A} \rightarrow \Omega^{U}{ }^{A}$ and $\mu_{M}^{U}: U_{M}^{2} \rightarrow U_{M}$ similarly.

In this section we only check the followings:

(i) $\zeta_{m}^{A}(f)$ is a primefilter for $m \in M$ and $f \in U_{M} A$.

Since $\sigma_{m}^{\#} \cdot \phi=\phi \notin f$, we have $\phi \notin \zeta_{m}^{A}(f)$. If $\alpha \cup \beta \in \zeta_{m}^{A}(f)$, then $\sigma_{m}^{\#} \cdot(\alpha \cup \beta)=$ $\left\langle\sigma_{m}^{\#} \cdot \alpha\right) \cup\left(\sigma_{m}^{\#} \cdot \beta\right) \in f$, so we have $\sigma_{m}^{\#} \cdot \alpha \in f$ or $\sigma_{m}^{\#} \cdot \beta \in f$. Therefore $\alpha \in \zeta_{m}^{A}(f)$ or $\beta \in \zeta_{m}^{A}(f)$.

(ii) $U_{M} \Phi(f)$ is a primefilter for $f \in U_{M} \boldsymbol{A}$.

It is similar to (i).

(iii) $\eta_{M}^{U} \boldsymbol{A}(a)$ is a primefilter for $a \in \boldsymbol{A}$.

$\phi \oplus \eta \boldsymbol{A}(\alpha)$ is trivial. If $\alpha \cup \beta \in \eta \boldsymbol{A}(a)$, then $(e, a) \in \alpha \cup \beta$, and so we have $(e, a) \in \alpha$ or $(e, a) \subseteq \beta$. Therefore $\alpha \in \eta \boldsymbol{A}(a)$ or $\beta \in \eta \boldsymbol{A}(a)$.

(iv) $\mu_{M}^{U} \boldsymbol{A}(\mathscr{I})$ is a primefilter for $\mathscr{I} \in U_{M}^{2} \boldsymbol{A}$.

Since $\pi_{M}^{U} \boldsymbol{A}(\dot{\phi})=\phi$, we have $\phi \oplus \mu \boldsymbol{A}(\mathscr{I})$. Then we obtain $\pi \boldsymbol{A}(\alpha \cup \beta)=\pi \boldsymbol{A}(\alpha) \cup \pi \boldsymbol{A}(\beta)$ from

$$
\begin{aligned}
& (m, f) \in \pi \boldsymbol{A}(\alpha \cup \beta) \\
& \leftrightarrow(\alpha \cup \beta) \cdot l_{m}=\left(\alpha \cdot l_{m}\right) \cup\left(\beta \cdot l_{m}\right) \in f \\
& \leftrightarrow \alpha \cdot l_{m} \in f \text { or } \beta \cdot l_{m} \in f \\
& \leftrightarrow(m, f) \in \pi \boldsymbol{A}(\alpha) \cup \pi \boldsymbol{A}(\beta) .
\end{aligned}
$$

If $\alpha \cup \beta \in \mu \boldsymbol{A}(\mathscr{T})$, then we have $\pi \boldsymbol{A}(\alpha \cup \beta)=\pi \boldsymbol{A}(\alpha) \cup \pi \boldsymbol{A}(\beta) \in \mathscr{F}$ and hence $\pi \boldsymbol{A}(\alpha) \in \mathscr{F}$ or $\pi \boldsymbol{A}(\beta) \in \mathscr{F}$. Therefore $\alpha \in \mu \boldsymbol{A}(\mathscr{F})$ or $\beta \in \mu \boldsymbol{A}(\mathscr{F})$.

We denote by $U=\left(U, \eta^{U}, \mu^{U}\right)$ the ultrafilter monad in Set [4].

Proposition 5.1. Let $\boldsymbol{A}$ be an $M$-set.

(a) For $f \in U_{M} \boldsymbol{A}, s_{F} \boldsymbol{A}(f)$ is a primefilter.

(b) If $M$ is a group, then $t_{F} \boldsymbol{A}(f)(f \in \bar{U} \boldsymbol{A})$ is a primefilter.

PROOF. (a) This is trivial by the definition of $s_{F}$.

(b) If $\alpha \cup \beta \in t_{F} A$, then $\bigcup_{a \in \alpha \cup \beta} \eta_{M}^{P} A(a)=\left(\bigcup_{a \in \alpha} \eta_{M}^{P}(a)\right) \cup\left(\bigcup_{b \in \beta} \eta_{M}^{P}(b)\right) \in f$, and so $\bigcup_{a \in \alpha} \eta_{M}^{P} \boldsymbol{A}(a) \in f$ or $\bigcup_{b \in \beta} \eta_{M}^{P} \boldsymbol{A}(b) \in f$. Therefore $\alpha \in t_{F} \boldsymbol{A}$ or $\beta \in t_{F} \boldsymbol{A}$.

If $M$ is a group, then we have $\Theta \in t_{F} \boldsymbol{A}(f)$, by $\bigcup_{a \in A} \eta_{M}^{P}(a)=M \times \boldsymbol{A}=\Theta \in f$.

By the last proposition we can define a natural transformation $s_{U}: \bar{U} \rightarrow U_{M}$ by by restricting a domain of $s_{F}$.

Now we can show the several properties of the primefilter monad by using the results of the filter monad.

THEOREM 5.2 .

(a) $\boldsymbol{U}_{M}=\left(U_{M}, \eta_{M}^{U}, \mu_{M}^{U}\right)$ is a monad in $M$-Set.

(b) If $M=1$, then $\boldsymbol{U}_{1}$ is the ultrafilter monad $\boldsymbol{U}$ in Set.

PROPOSITION 5.3. The natural transformation $s_{U}: \bar{U}_{\rightarrow} \rightarrow U_{M}$ is algebraic.

THEOREM 5.4. If $M$ is a group, then the algebraic functor $s_{U}^{*}: M$-Set ${ }_{M \rightarrow M}^{U_{M}}$ Set $^{\bar{U}}$ is an isomorphism of categories.

We denote by $\mathrm{CH}$ the category of compact Hausdorff spaces and continuous maps.

COROLlary 5.5. If $M$ is a group, then

$$
M-\operatorname{Set}^{U} M \cong-\left(\mathrm{Set}^{U}\right) \cong M-\mathrm{CH} .
$$


If $M$ is a group then, by the last corollary, we can identify a $U_{M}$-algebra with a compact Hausdorff space equipped with a continuous $M$-action.

\section{Acknowledgements}

The author would like to express his sincere thanks to Professor Yasuo Kawahara for his valuable suggestions, constructive comments and encouragement during the course of this study. The author wishes to thank Dr. Satoru Miyano for his critical reading the manuscript and helpful comments.

\section{References}

[1] A. DAY: Filter monads, continuous lattices and closure systems. Can. J. Math., 27 (1975), 50-59.

[2] R. Goldblatt : Topoi, The categorial analysis of logic. North-Holland, Amsterdam, (1979).

[3] S. MAC LANE: Categories for the working mathematician. Springer-Verlag, New York, (1971).

[4] E. G. MANEs: Algebraic theories. Springer-Verlag, New York, (1976).

[5] D. ScotT: Continuous lattices. Lecture Notes in Math., 274 (1972), 93-136.

[6] O. WYLER: Algebraic theories of continuous lattices. Lecture Notes in Math., 871 (1981), $390-413$.

Communicated by S. Arikawa

Received September 29, 1984 